\title{
Chemotherapy Effectiveness and Prognosis of Gastric Cancer Influenced by PTPN11 Polymorphisms
}

\author{
Chuanjun Zhuo a,b,c,d Mingjing Shao ${ }^{e}$ Ce Chen ${ }^{c}$ Chongguang Lin ${ }^{c}$ Deguo Jiang ${ }^{c}$ \\ Guangdong Chen ${ }^{c}$ Hongjun Tian ${ }^{a}$ Lina Wang $^{a}$ Jie Lia Xiaodong Lin ${ }^{c}$ \\ ${ }^{a}$ Tianjin Anding Hospital, Tianjin, ${ }^{b}$ Tianjin Anning Hospital, Tianjin, 'Wenzhou Seventh People's Hospital, \\ Wenzhou, Zhejiang, 'Institute of Mental Health, Jining Medical University, Jining, Shandong, ${ }^{~}$ China- \\ Japan Friendship Hospital, Beijing, China
}

\section{Key Words}

Gastric cancer • PTPN11 • SNP • Chemotherapy •Prognosis • Gene-environment interaction

\begin{abstract}
Objective: Since gastric cancer (GC) cells exhibited higher grades of SHP-2 encoded by PTPN11 than normal cells, it would be intriguing to explore whether PTPN11 single nucleotide polymorphisms (SNPs) would influence chemotherapy effectiveness and GC prognosis among a Chinese population. Methods: Altogether 430 late-stage GC patients and 960 healthy controls matched with age and sex were incorporated. Three PTPN11 SNPs (i.e. rs7958372, rs12229892 and rs2301756) were genotyped by polymerase chain reaction-restriction fragment length polymorphism (PCR-RFLP). Chemotherapies of cisplatin and 5-fluorouracil were performed for 4 cycles. Odds ratios (ORs) and 95\% confidence intervals (CIs) were calculated using the logistic regression. Survival curves were plotted with Kaplan-Meier method and the COX proportional hazard model was used to analyze independent factors for GC prognosis. Results: For rs12229892, AA and GA genotypes would cause 1.60-fold increase of GC risk in comparison to homozygote GG (OR = 1.60; 95\% CI = 1.23-2.07; $P<0.001)$. The A allele of rs2301756 was significantly associated with a decrease in the risk of GC when compared with $G$ allele (OR $=0.81 ; 95 \% \mathrm{CI}=0.65-0.99 ; P=0.043)$. Results from both 2 -cycle and 4-cycle chemotherapy suggested that chemotherapy was significantly more effective for GA and AA genotypes of rs2301756 compared with homozygote GG $(P<0.001)$. Besides, the joint impact of rs 12229892 (AA) and environmental factors (i.e. smoking, family history, intake of processed food and $H$ .pylori infection) on GC risk was considered as positive interaction, while that of rs2301756 (AA) and the above parameters was deemed as negative interaction. Finally, differentiation degree, axillary lymph node metastasis, rs12229892 and rs2301756 appeared as independent risk factors for GC development (all $P<0.05$ ). Conclusion: Since rs2301756 polymorphism of PTPN11 was associated with reduced risk of GC and better effects of chemotherapy on GC, it can be considered as a predictor of GC prognosis and the treatment target for GC.

C. Zhuo, M. Shao and C. Chen contributed equally to this work as co-first author.

Xiaodong Lin, Wenzhou Seventh People's Hospital, No. 552 East Xishan Road, Ouhai District,

Wenzhou, Zhejiang, 325005, (China)

Tel. +86 0577-88209928. Fax +86 0577-88432238. E-Mail lidongww@yeah.net 


\section{Cellular Physiology Cell Physiol Biochem 2016;39:1537-1552 \\ \begin{tabular}{ll|l} 
and Biochemistry & $\begin{array}{l}\text { DOI: 10.1159/000447856 } \\
\text { Published online: September 12, } 2016\end{array}$ & $\begin{array}{l}\text { C } 2016 \text { The Author(s). Published by S. Karger AG, Basel } \\
\text { www.karger.com/cpb }\end{array}$ \\
\hline
\end{tabular} \\ Zhuo et al.: PTPN11 Polymorphisms and GC}

\section{Introduction}

Gastric cancer (GC) is one common malignancy with high incidence and mortality in Eastern Asia, and the incidence of GC in males is nearly two times higher than that in females $[1,2]$. To date, GC is divided into intestinal and diffuse types based on histopathological classification: the former type is usually environment-induced and featured by cells with irregular tubular structures, while the latter type is mainly triggered by genetic factors (e.g. E-cadherin mutations) and characterized by poorly-differentiated cells that produce nonadhesive and secreted mucus [3] . As was suggested by several researchers, Helicobacter pylori (H. pylori) has been identified as a chief risk factor for GC development as it induces gastric atrophy and develops precancerous lesions [4, 5]. Meanwhile, the degree of gastric damage induced by $H$. pylori infection seemed to vary among individuals, suggesting that the host genetic traits could play key roles in the development of gastritis and even GC [6-8].

One gene of interest appeared as tyrosine-protein phosphatase non-receptor 11 (PTPN11), which encodes Src homology 2 domain-containing protein tyrosine phosphatase (SHP-2) within gastric epithelial cells [9]. The binding of SHP-2 to cytoxin-associated gene A (CagA) of $H$. pylori would facilitate formation of a scattering phenotype, the so-called hummingbird phenotype, through stimulating phosphatase activities of SHP-2 and thereby extending activation of Erk $[10,11]$. The hummingbird shape has been commonly recognized as a cellular alteration that symbolizes gastritis, which was identified as the previous stage of gastric carcinogenesis $[11,12]$. The key role of SHP-2 in GC development was also certified with evidence that functional polymorphisms of PTPN11 could modulate several signaling pathways, including mitogenic activation, cell migration and metabolic control, in patients with $H$. pylori infection and that GC cells exhibited higher grades of SHP-2 than normal cells $[10,13]$.

Apart from GC development, GC prognosis was also noteworthy. The 5-year survival rate of GC remained depressingly low, since that GC usually could not be diagnosed until in its advanced stages [14], during which period GC progression can merely be controlled, yet not cured, by either adjuvant radiotherapy or chemotherapy $[15,16]$. Notably, variations of genes (e.g. GSTP1 and VEGF) seemed to confer GC patients distinct prognoses and resistances to fluoropyrimidine-/platinum- based chemotherapies [17]. For instance, for advanced GC patients, subjects carrying $\geq 1{ }^{105} \mathrm{Val}$ allele of GSTP1 were predisposed to enjoy superior prognosis and effective responses to regimens based on oxaliplatin/5-FU [16]. Therefore, it was hypothesized that identification of PTPN11 polymorphisms may help predict GC prognosis after chemotherapies.

To validate the above hypothesis, this study was specifically designated to assess whether single-nucleotide polymorphisms (SNPs) of PTPN11 are associated with both GC development and chemotherapy effects on GC. Since rs2301756 which is located in intron3 was connected with elevated risk of gastric atrophy among a Japanese population with $H$. pylori infection, several rs2301756-centered tag SNPs were arranged for this study [18]. Moreover, environmental factors that might also account for GC development were also researched on, including smoking, family history, intake of processed food and Helicobacter pylori (H. pylori) infection.

\section{Materials and Methods}

\section{Study subjects}

In total, 1390 subjects were incorporated in this study, including 430 patients with GC (case group) and 960 healthy subjects (control group). The case group consisted of 314 males and 116 females who were diagnosed with late-stage GC between March 2007 and March 2011. The average age of the case group was $62.75 \pm 11.40$ years old. All GC patients in the case group were selected according to the following criteria: (1) late-stage GC confirmed by pathology or cytology; (2) patients had at least 1 lesion measurement confirmed by CT; (3) normal bone marrow function and key normal organ (e.g. liver, kidney, heart, lung) functions were 


\section{Cellular Physiology Cell Physiol Biochem 2016;39:1537-1552 \begin{tabular}{ll|l} 
and Biochemistry & $\begin{array}{l}\text { DOI: 10.1159/000447856 } \\
\text { Published online: September 12, } 2016\end{array}$ & $\begin{array}{l}\text { @ } 2016 \text { The Author(s). Published by S. Karger AG, Basel } \\
\text { www.karger.com/cpb }\end{array}$ \\
\hline
\end{tabular} Zhuo et al.: PTPN11 Polymorphisms and GC}

retained; (4) No chemotherapy program had been performed one-month prior to the inclusion of patients. All subjects were independent Han Chinese and there was no consanguinity identified among the subjects. Besides that, 960 cases of healthy individuals ( 609 males and 351 females) were incorporated in the control group and they were selected during the same period with an average age of $63.26 \pm 10.50$ years old. All subjects have signed the informed consent form and this research was approved by the Ethics Committee of Wenzhou Seventh People's Hospital.

\section{Identification of PTPN11 polymorphisms}

Specimen collection and DNA extraction: we extracted $2 \mathrm{ml}$ peripheral venous blood from both fasting patients and healthy subjects into tubes with ethylene diamine tetraacetic acid (EDTA) anticoagulant, then they were stored at $-20^{\circ} \mathrm{C}$; DNA extraction Kit (United States Promega Corporation) was applied to extract DNA according to the protocols and the extracted DNA were stored at $-80^{\circ} \mathrm{C}$.

Primer Design and synthesis: As suggested by the target gene sequence and the selection of polymorphic loci, the Sequenom Mass ARRAY @Assay Design 3.1 software (United States Sequenom Company) was used to design multiple PCR amplification primers and single base extension primers and the synthesis of these primers were performed by Shanghai Chun Biological Company (Invitrogen). In order to identify PTPN11 gene polymorphism of rs7958372, rs12229892 and rs2301756, the method of polymerase chain reactionrestriction fragment length polymorphism (PCR-RFLP) was utilized in different actions with primers shown in Table 1. The PCR amplification system was set as follows: $100 \mathrm{ng}$ genomic DNA, $1.5 \mathrm{ul}$ of Taq polymerase, $25 \mathrm{mmol}$ of dNTP and $2.5 \mathrm{pmol}$ of PCR primers. PCR reaction conditions were set as follows: stage I, predegenerated PCR cycling at $95^{\circ} \mathrm{C}$ for 4 minutes, then 35 cycles of 45 seconds at $95^{\circ} \mathrm{C}, 30$ seconds at $5-6^{\circ} \mathrm{C}$ and 30 seconds at $72^{\circ} \mathrm{C}$, extension with 7 minutes at $72^{\circ} \mathrm{C}$; stage II, PTPN11 (rs7958372, rs12229892, rs2301756) synthesis, PCR cycling at $94^{\circ} \mathrm{C}$ for 5 minutes, then 35 cycles of 45 seconds at $94^{\circ} \mathrm{C}, 45$ seconds at $60^{\circ} \mathrm{C}$ and 30 seconds at $72^{\circ} \mathrm{C}$, extension with 5 minutes at $72^{\circ} \mathrm{C}$. After that, $5 \mu \mathrm{l}$ of PCR products was taken and incubated with restriction endonucleases overnight and the temperature was set to 65 degrees centigrade. The amplified DNA were electrophoresed in the 3\% agarose gel, stained with ethidium bromide, then scanned using ultraviolet light transilluminator and finally images were obtained and analyzed.

\section{Chemotherapy}

Chemotherapy of cisplatin $\left(20 \mathrm{mg} / \mathrm{m}^{2}\right.$, intravenous drip, from day 1 to day 4 , then withdrawal, regained from day 25 to 28$)$ and 5 -fluorouracil $\left(750 \mathrm{mg} / \mathrm{m}^{2}\right.$, intravenous drip, from day 1 to day 4 , then withdrawal, regained from day 25 to 28 ) were performed for 4 cycles. Then the chemotherapy effects on solid tumors were evaluated every two cycles based on the WHO clinical solid tumor evaluation standards.

Based on response evaluation criteria in solid tumors (RECIST), around 4 evaluation standards were identified in the present study, namely, complete response (CR), partial response (PR), stable disease (SD) and progressive disease (PD). Their definitions were enlisted as follows: CR, all target lesions disappeared; $\mathrm{PR}$, the sum of baseline long diameters shortened by $\geq 30 \%$; SD, change of the sum of baseline long diameters was between PR and SD; PD, the sum of baseline long diameters lengthened by $\geq 20 \%$ or new lesions appeared.

\section{Follow-up period}

We designed questionnaires and collected all relevant information in order to assess the survival status of 430 patients with GC. All relevant information was collected by mail and telephone up to January 2015. Overall survival was calculated from date of post-chemotherapy to the deadline of the follow-up period or date of death. The expiration date was defined as postmortem interval (PMI) because of GC or last follow-up day. And for GC patients who died of other causes, their survival time was recorded as censored data.

Table 1. Primer sequences of PTPN11 polymorphisms

\begin{tabular}{lll}
\hline SNP & Sense & Anti-sense \\
\hline rs12229892 & 5'-GAATGAATGGTGCTGCTAC-3' & 5'-CTGGGATTACAGGCGTGAG-3' \\
rs7958372 & 5'-CCCCTATGTGCCCTATTGT-3' & 5'-CTGGGATTACAGGCGTGAG-3' \\
rs2301756 & 5'-TTTAGCTCTACTATGGGTTCA-3' & 5'-TTCCAAAGTGTTGGGATTA-3' \\
\hline
\end{tabular}



Cellular Physiology Cell Physiol Biochem 2016;39:1537-1552 \begin{tabular}{ll|l|l} 
DOI: 10.1159/000447856 & 02016 The Author(s). Published by S. Karger AG, Basel \\
\hline
\end{tabular} and Biochemistry Published online: September 12, 2016 www.karger.com/cpb
Zhuo et al.: PTPN11 Polymorphisms and GC

Table 2. Clinical characteristics of gastric cancer patients and healthy controls included in our study

\begin{tabular}{|c|c|c|c|}
\hline Characteristics & Control $(n=960)$ & Case $(n=430)$ & $P$ value \\
\hline Age (yrs) & $63.26 \pm 10.50$ & $62.75 \pm 11.40$ & 0.415 \\
\hline \multicolumn{4}{|l|}{ Sex } \\
\hline Female & $351(36.56 \%)$ & $156(36.28 \%)$ & \multirow[t]{2}{*}{0.952} \\
\hline Male & $609(63.44 \%)$ & $274(63.72 \%)$ & \\
\hline \multicolumn{4}{|l|}{ Smoking status } \\
\hline Yes & $425(44.27 \%)$ & $228(53.02 \%)$ & \multirow[t]{2}{*}{0.003} \\
\hline No & $535(55.73 \%)$ & $202(46.98 \%)$ & \\
\hline \multicolumn{4}{|l|}{ Family history } \\
\hline Yes & $143(14.90 \%)$ & $109(25.35 \%)$ & \multirow[t]{2}{*}{$<0.001$} \\
\hline No & $817(85.10 \%)$ & $321(74.65 \%)$ & \\
\hline \multicolumn{4}{|l|}{ Intake of processed food } \\
\hline$<8$ kilograms/year & $458(47.71 \%)$ & $287(66.74 \%)$ & \multirow[t]{2}{*}{$<0.001$} \\
\hline$\geq 8$ kilograms/year & $502(52.29 \%)$ & $143(33.26 \%)$ & \\
\hline \multicolumn{4}{|l|}{ H. pylori infection } \\
\hline Positive & $472(49.17 \%)$ & $300(69.77 \%)$ & \multirow[t]{2}{*}{$<0.001$} \\
\hline Negative & $488(50.83 \%)$ & $130(30.23 \%)$ & \\
\hline \multicolumn{4}{|l|}{ Pathological subtype } \\
\hline Adenocarcinoma & & $304(70.70 \%)$ & \\
\hline Mucoid carcinoma & & $37(8.60 \%)$ & \\
\hline Signet-ring cell carcinoma & & $49(11.39 \%)$ & \\
\hline Others & & $40(9.31 \%)$ & \\
\hline \multicolumn{4}{|l|}{ Differentiation degree } \\
\hline Well differentiated & & $14(3.26 \%)$ & \\
\hline Moderately differentiated & & $167(38.84 \%)$ & \\
\hline Poorly differentiated & & $222(51.63 \%)$ & \\
\hline Others & & $27(6.27 \%)$ & \\
\hline \multicolumn{4}{|l|}{ Clinical stages } \\
\hline IIIA & & $13(3.02 \%)$ & \\
\hline IIIB & & $51(11.86 \%)$ & \\
\hline IIIC & & $225(52.33 \%)$ & \\
\hline IV & & $141(32.79 \%)$ & \\
\hline \multicolumn{4}{|l|}{ Axillary lymph node metastasis } \\
\hline $\mathrm{N}_{0-1}$ & & $79(18.37 \%)$ & \\
\hline $\mathrm{N}_{2-3}$ & & $351(91.63 \%)$ & \\
\hline \multicolumn{4}{|l|}{ CEA } \\
\hline$<5 \mathrm{ng} / \mathrm{mL}$ & & $255(58.30 \%)$ & \\
\hline$\geq 5 \mathrm{ng} / \mathrm{mL}$ & & $175(41.70 \%)$ & \\
\hline
\end{tabular}

Statistical analysis

Age was expressed in the form of mean \pm standard deviation and compared using the Student's t-test. Categorical data were analyzed using the Chi-square test. The Hardy-Weinberg equilibrium (HWE) test was used to assess whether the genotype frequency in the control group was complied with the expected frequency which were evaluated using the Hardy-Weinberg Equilibrium Theory $(p=$ allele frequency, $q=$ $\left.1-p, p^{2}+q^{2}=1\right)$. Differences in genotype and allele frequency between the control and case group were evaluated using the $\chi^{2}$ test. Odds ratios (ORs) and 95\% confidence intervals (CI) adjusting for gender and age were calculated using the method of logistic regression. Type-II gene-environment interaction (GEI) model was applied to analyze interactive effects of genetic and relevant environmental factors on GC development. And we judged whether the interaction reactions existed and which type the gene-environment action 

Cellular Physiology Cell Physiol Biochem 2016;39:1537-1552 \begin{tabular}{ll|l} 
DOI: 10.1159/000447856 & O 2016 The Author(s). Published by S. Karger AG, Basel \\
and Biochemistry Published online: Septer 12,2016 &
\end{tabular} Zhuo et al.: PTPN11 Polymorphisms and GC

Table 3. Association of PTPN11 SNPs (rs12229892, rs7958372 and rs2301756) with development of gastric cancer risk

\begin{tabular}{|c|c|c|c|c|c|}
\hline Genotype & Case $(n, \%)$ & Control (n, \%) & OR & $95 \% \mathrm{CI}$ & $P$ value \\
\hline \multicolumn{6}{|c|}{ rs12229892 } \\
\hline \multicolumn{6}{|l|}{ Genotype } \\
\hline GG & $104(24.19 \%)$ & $324(33.75 \%)$ & Reference & & \\
\hline GA & $255(59.30 \%)$ & $455(47.40 \%)$ & 1.75 & $1.33-2.29$ & $<0.001$ \\
\hline $\mathrm{AA}$ & $71(16.51 \%)$ & $181(18.85 \%)$ & 1.22 & $0.86-1.74$ & 0.264 \\
\hline $\mathrm{GA}+\mathrm{AA}$ & $326(75.81 \%)$ & $636(66.25 \%)$ & 1.60 & $1.23-2.07$ & $<0.001$ \\
\hline \multicolumn{6}{|l|}{ Allele } \\
\hline G & 463 (53.84\%) & $1103(57.45 \%)$ & Reference & & \\
\hline A & $397(46.16 \%)$ & $817(42.55 \%)$ & 1.16 & $0.98-1.36$ & 0.076 \\
\hline \multicolumn{6}{|l|}{ rs7958372 } \\
\hline \multicolumn{6}{|l|}{ Genotype } \\
\hline TT & $317(73.72 \%)$ & $712(74.17 \%)$ & Reference & & \\
\hline TC & $106(24.65 \%)$ & $231(24.06 \%)$ & 1.03 & $0.79-1.34$ & 0.824 \\
\hline $\mathrm{CC}$ & $7(1.63 \%)$ & $16(1.67 \%)$ & 0.98 & $0.40-2.41$ & 0.969 \\
\hline $\mathrm{TC}+\mathrm{CC}$ & $113(26.28 \%)$ & $247(25.73 \%)$ & 1.02 & $0.79-1.33$ & 0.837 \\
\hline \multicolumn{6}{|l|}{ Allele } \\
\hline $\mathrm{T}$ & $740(86.05 \%)$ & $1655(86.20 \%)$ & Reference & & \\
\hline C & $120(13.95 \%)$ & $263(13.80 \%)$ & 1.02 & $0.81-1.29$ & 0.865 \\
\hline \multicolumn{6}{|l|}{ rs2301756 } \\
\hline \multicolumn{6}{|l|}{ Genotype } \\
\hline GG & $295(68.60 \%)$ & $634(66.04 \%)$ & Reference & & \\
\hline $\mathrm{GA}$ & $123(28.60 \%)$ & $261(27.19 \%)$ & 1.01 & $0.78-1.31$ & 0.922 \\
\hline $\mathrm{AA}$ & $12(2.80 \%)$ & $65(6.77 \%)$ & 0.39 & $0.21-0.75$ & 0.003 \\
\hline $\mathrm{GA}+\mathrm{AA}$ & 135 (33.95\%) & $326(33.96 \%)$ & 0.89 & $0.69-1.14$ & 0.348 \\
\hline \multicolumn{6}{|l|}{ Allele } \\
\hline G & 713 (82.91\%) & $1529(79.64 \%)$ & Reference & & \\
\hline A & $147(17.09 \%)$ & $391(20.36 \%)$ & 0.81 & $0.65-0.99$ & 0.043 \\
\hline
\end{tabular}

belonged to base on interaction coefficient $\left(\gamma, \gamma=\beta_{\mathrm{eg}} / \beta_{\mathrm{e}}\right)$ [19]. Survival curve was plotted using the Kaplan Meier method and comparison of survival rate was evaluated by the Log-Rank test. The Cox Proportional Hazards model was used to assess independent factors for GC prognosis. P values $<0.05$ were considered as statistical significant. Data were analyzed with SPSS Software package19.0 (SPSS Inc., Chicago, IL, USA).

\section{Results}

Clinical characteristics of GC patients and healthy controls

GC patients and healthy controls were well matched in age $(P=0.415)$ and sex ratio $(P=0.952$; Table 2). Such environmental hazards as smoking status, family history, intake of processed food and H. pylori infection all imposed significant impacts on susceptibility to GC $(P<0.05)$. Moreover, the pathological types of GC samples were primarily made up of adenocarcinoma $(70.70 \%)$, mucoid carcinoma $(8.60 \%)$ and signet-ring cell carcinoma $(11.39 \%)$, and the prevalence of patients with well-, moderately- and poorly- differentiated GC appeared as 3.26\%, 38.84\% and 51.63\%, respectively. Additionally, in accordance with GC tumor node metastasis (TNM) staging criteria enacted by union for international cancer control/American joint committee on cancer (UICC/AJCC), 430 GC patients were categorized into IIIA (3.02\%), IIIB (11.86\%), IIIC (52.33\%) and IV (32.79\%) clinical stages [20]. The 


\section{Cellular Physiology Cell Physiol Biochem 2016;39:1537-1552 \begin{tabular}{ll|l} 
DOI: 10.1159/000447856 & $\begin{array}{l}\text { O 2016 The Author(s). Published by S. Karger AG, Basel } \\
\text { www.karger.com/cpb }\end{array}$ \\
\hline
\end{tabular} \\ Zhuo et al.: PTPN11 Polymorphisms and GC}

Table 4. Association of PTPN11 SNPs (rs12229892, rs7958372 and rs2301756) with chemotherapy responses of gastric cancer

\begin{tabular}{|c|c|c|c|c|c|c|c|c|c|c|c|}
\hline \multirow{2}{*}{ SNPs } & \multirow{2}{*}{$\mathrm{N}$} & \multicolumn{2}{|c|}{ Four cycles } & \multirow{2}{*}{ OR } & \multirow{2}{*}{$95 \% \mathrm{Cl}$} & \multirow{2}{*}{$P$ value ${ }^{*}$} & \multicolumn{2}{|c|}{ Two cycles } & \multirow{2}{*}{ OR } & \multirow{2}{*}{$95 \% \mathrm{Cl}$} & \multirow{2}{*}{$P$ value ${ }^{*}$} \\
\hline & & $C R+P R$ & $S D+P D$ & & & & $C R+P R$ & $S D+P D$ & & & \\
\hline \multicolumn{12}{|c|}{ rs12229892 } \\
\hline GG & 104 & 46 & 58 & & & Reference & 47 & 57 & & & Reference \\
\hline GA & 255 & 134 & 121 & 0.97 & $0.71-1.36$ & 0.153 & 138 & 117 & 0.70 & $0.44-1.11$ & 0.125 \\
\hline $\mathrm{AA}$ & 71 & 39 & 32 & 1.08 & $0.64-1.81$ & 0.164 & 42 & 29 & 0.57 & $0.31-1.05$ & 0.070 \\
\hline $\mathrm{GA}+\mathrm{AA}$ & 326 & 173 & 153 & 0.70 & $0.45-1.09$ & 0.117 & 170 & 156 & 0.76 & $0.49-1.18$ & 0.217 \\
\hline \multicolumn{12}{|l|}{ rs7958372 } \\
\hline TT & 317 & 150 & 167 & & & Reference & 142 & 175 & & & Reference \\
\hline TC & 106 & 59 & 47 & 0.72 & $0.46-1.11$ & 0.137 & 55 & 50 & 0.74 & $0.47-1.15$ & 0.177 \\
\hline CC & 7 & 4 & 3 & 0.67 & $0.15-3.06$ & 0.607 & 4 & 4 & 0.81 & $0.20-3.30$ & 0.770 \\
\hline $\mathrm{TC}+\mathrm{CC}$ & 113 & 63 & 50 & 0.71 & $0.46-1.10$ & 0.124 & 59 & 54 & 0.74 & $0.48-1.14$ & 0.175 \\
\hline \multicolumn{12}{|c|}{ rs2301756 } \\
\hline GG & 284 & 110 & 174 & & & Reference & 92 & 192 & & & Reference \\
\hline GA & 123 & 108 & 15 & 0.09 & $0.05-0.16$ & $<0.001$ & 101 & 30 & 0.14 & $0.09-0.23$ & $<0.001$ \\
\hline $\mathrm{AA}$ & 12 & 10 & 2 & 0.13 & $0.03-0.59$ & 0.002 & 11 & 4 & 0.17 & $0.05-0.56$ & 0.001 \\
\hline $\mathrm{GA}+\mathrm{AA}$ & 146 & 128 & 18 & 0.09 & $0.05-0.15$ & $<0.001$ & 112 & 34 & 0.14 & $0.09-0.23$ & $<0.001$ \\
\hline
\end{tabular}

Table 5 The layer analysis on interaction between genotypes of PTPN11 SNPs (i.e. rs12229892 and rs2301756) and susceptibility to gastric cancer

\begin{tabular}{cllllll}
\hline \multicolumn{2}{c}{ Genotype } & Case (n, \%) & Control (n, \%) & OR & $95 \%$ CI & P value \\
rs12229892 & rs2301756 & & & & & \\
\hline GG & GG & $71(16.51 \%)$ & $214(22.30 \%)$ & 0.69 & $0.51-0.93$ & 0.014 \\
GG & GA & $30(6.98 \%)$ & $88(9.17 \%)$ & 0.74 & $0.48-1.14$ & 0.176 \\
GG & AA & $3(0.70 \%)$ & $22(2.29 \%)$ & 0.30 & $0.09-1.01$ & 0.039 \\
GA & GG & $175(40.70 \%)$ & $301(31.35 \%)$ & 1.50 & $1.19-1.90$ & $<0.001$ \\
GA & GA & $73(16.98 \%)$ & $124(12.92 \%)$ & 1.38 & $1.01-1.89$ & 0.045 \\
GA & AA & $7(1.63 \%)$ & $31(3.23 \%)$ & 0.49 & $0.22-1.23$ & 0.087 \\
AA & GG & $49(11.40 \%)$ & $120(12.50 \%)$ & 0.83 & $0.59-1.19$ & 0.314 \\
AA & GA & $20(4.65 \%)$ & $49(5.10 \%)$ & 0.91 & $0.53-1.55$ & 0.719 \\
AA & AA & $2(0.47 \%)$ & $12(1.25 \%)$ & 0.37 & $0.08-1.66$ & 0.176 \\
\hline
\end{tabular}

number of GC patients diagnosed as N2-3 axillary lymph node metastasis was nearly 3.44 folds more than that confirmed as N0-1 node metastasis. Furthermore, GC populations with in vivo carcinoembryonic antigen (CEA) contents of $<5 \mathrm{ng} / \mathrm{mL}$ and $\geq 5 \mathrm{ng} / \mathrm{mL}$ were roughly equal in size (58.30\% vs. $41.70 \%)$.

Difference in PTPN11 SNP genotype distributions and allele frequencies

Genotype frequencies of rs12229892, rs7958372 and rs2301756 in the control group all complied with HWE (all $P>0.05$ ). As for rs12229892 (Table 3), subjects carrying heterozygote GA were more prone to develop GC than ones carrying homozygote GG (OR $=1.75,95 \% \mathrm{CI}=1.33-2.29, P<0.001)$. The dominant model also implied that genotypes $\mathrm{GA}+\mathrm{AA}$ were at greater risk of $\mathrm{GC}$ than homozygote $\mathrm{GG}(\mathrm{OR}=1.60,95 \% \mathrm{CI}=1.23-2.07, P$ $<0.001$ ). Besides, it could be drawn from the allelic model of rs2301756 (Table 3) that GC patients possessed notably lower frequency of $A$ allele than that of $G$ allele $(O R=0.81,95 \%$ $\mathrm{CI}=0.65-0.99, P=0.043)$. And carriers with homozygote AA appeared to suffer from GC less readily than those with genotype GG $(\mathrm{OR}=0.39,95 \% \mathrm{CI}=0.21-0.75, P=0.003)$. However, with respect to rs7958372, no statistically significant distinctions were observed in both genotype and allele frequencies between GC patients and healthy controls (all $P>0.05$ ). 

Cellular Physiology Cell Physiol Biochem 2016;39:1537-1552 \begin{tabular}{ll|l|l|l|l}
\hline DOI: $10.1159 / 000447856$ & 2016 The Author(s). Published by S. Karger AG, Basel
\end{tabular} and Biochemistry Published online: September 12, 2016 www.karger.com/cpb
Zhuo et al.: PTPN11 Polymorphisms and GC

Table 6. The conditional logistic regression analysis on interaction between PTPN11 SNPs (i.e. rs12229892 and rs2301756) and smoking status

\begin{tabular}{lllllllll}
\hline Genotype & Smoking & Case (n, \%) & Control (n, \%) & OR & $95 \% \mathrm{CI}$ & $\beta_{e}$ & $\beta_{\text {eg }}$ & $\gamma$ \\
\hline rs12229892 & & & & & & & & \\
GG & No & $49(11.39 \%)$ & $181(18.85 \%)$ & & & & & \\
GA & No & $120(27.91 \%)$ & $254(26.46 \%)$ & 1.75 & $1.19-2.56$ & & & \\
AA & No & $33(7.67 \%)$ & $101(10.52 \%)$ & 1.21 & $0.73-2.00$ & & & \\
GG & Yes & $55(12.79 \%)$ & $143(14.89 \%)$ & 1.42 & $0.91-2.21$ & 0.35 & & \\
GA & Yes & $135(31.40 \%)$ & $201(20.94 \%)$ & 2.48 & $1.69-3.64$ & 0.35 & 0.91 & 2.59 \\
AA & Yes & $38(8.84 \%)$ & $80(8.34 \%)$ & 1.76 & $1.07-2.89$ & 0.35 & 0.57 & 1.61 \\
rs2301756 & & & & & & & & \\
GG & No & $139(32.33 \%)$ & $353(36.77 \%)$ & & & & & \\
GA & No & $58(13.49 \%)$ & $145(15.10 \%)$ & 1.02 & $0.71-1.46$ & & & \\
AA & No & $6(1.39 \%)$ & $36(3.75 \%)$ & 0.42 & $0.17-1.03$ & & & \\
GG & Yes & $156(36.28 \%)$ & $281(29.27 \%)$ & 1.41 & $1.07-1.86$ & 0.34 & & \\
GA & Yes & $65(15.12 \%)$ & $116(12.08 \%)$ & 1.42 & $0.99-2.04$ & 0.34 & 0.35 & 1.02 \\
AA & Yes & $6(1.39 \%)$ & $29(3.02 \%)$ & 0.53 & $0.21-1.25$ & 0.34 & -0.63 & -1.84 \\
\hline
\end{tabular}

Table 7. The conditional logistic regression analysis on interaction between PTPN11 SNPs (i.e. rs12229892 and rs2301756) and family history

\begin{tabular}{|c|c|c|c|c|c|c|c|c|}
\hline Genotype & Family history & Case $(n, \%)$ & Control $(\mathrm{n}, \%)$ & OR & $95 \% \mathrm{CI}$ & $\beta_{c}$ & $\beta_{e g}$ & $\gamma$ \\
\hline \multicolumn{9}{|c|}{ rs12229892 } \\
\hline GG & No & $78(18.14 \%)$ & $276(28.75 \%)$ & & & & & \\
\hline GA & No & $190(44.19 \%)$ & 387 (40.31\%) & 1.74 & $1.28-2.36$ & & & \\
\hline $\mathrm{AA}$ & No & $53(12.33 \%)$ & $154(16.04 \%)$ & 1.22 & $0.82-1.81$ & & & \\
\hline GG & Yes & $26(6.05 \%)$ & $48(5.00 \%)$ & 1.92 & $1.12-3.28$ & 0.65 & & \\
\hline GA & Yes & $65(15.12 \%)$ & $68(7.08 \%)$ & 3.38 & $2.22-5.16$ & 0.65 & 1.22 & 1.87 \\
\hline $\mathrm{AA}$ & Yes & $18(4.17 \%)$ & $27(2.82 \%)$ & 2.36 & $1.23-4.51$ & 0.65 & 0.86 & 1.32 \\
\hline \multicolumn{9}{|l|}{ rs2301756 } \\
\hline GG & No & $220(51.16 \%)$ & $540(56.25 \%)$ & & & & & \\
\hline GA & No & $92(21.39 \%)$ & $222(23.13 \%)$ & 1.02 & $0.76-1.36$ & & & \\
\hline $\mathrm{AA}$ & No & $9(2.09 \%)$ & $55(5.73 \%)$ & 0.40 & $0.20-0.83$ & & & \\
\hline GG & Yes & $75(17.44 \%)$ & $94(9.79 \%)$ & 1.96 & $1.39-2.76$ & 0.67 & & \\
\hline GA & Yes & $31(7.21 \%)$ & $39(4.06 \%)$ & 1.95 & $1.19-3.21$ & 0.67 & 0.66 & 0.99 \\
\hline AA & Yes & $3(0.71 \%)$ & $10(1.04 \%)$ & 0.74 & $0.20-2.70$ & 0.67 & -0.30 & -0.44 \\
\hline
\end{tabular}

Association between PTPN11 polymorphisms and GC patients' response to chemotherapy

Either heterozygote GA or homozygote AA was tightly correlated with favorable shortterm efficacy of chemotherapy (CR+PR) in comparison with homozygote GG, whether 4-cycle $(\mathrm{GA}: \mathrm{OR}=0.09,95 \% \mathrm{CI}=0.05-0.16, P<0.001 ; \mathrm{AA}: \mathrm{OR}=0.13,95 \% \mathrm{CI}=0.03-0.59, P=0.002)$ or 2-cycle chemotherapeutic responses (GA: $\mathrm{OR}=0.14,95 \% \mathrm{CI}=0.09-0.23, P<0.001$; AA: $\mathrm{OR}=0.17,95 \% \mathrm{CI}=0.05-0.56, P=0.001$ ) were considered (Table 4 ). Meanwhile, results of both 4-cycle and 2-cycle chemotherapies indicated that the effective rate for GA+AA of rs2301756 was significantly higher than that for GG genotype with ORs of 0.09 (95\% CI = $0.05-0.16, P<0.001)$ and $0.14(95 \% \mathrm{CI}=0.09-0.23, P<0.001)$, respectively (Table 4). No significant associations were observed between rs12229892 or rs7958372 and effects of 2-cycle/4-cycle chemotherapy on GC patients. 


\section{Cellular Physiology Cell Physiol Biochem 2016;39:1537-1552 \begin{tabular}{l|l|l} 
DOI: 10.1159/000447856 & (c) 2016 The Author(s). Published by S. Karger AG, Basel
\end{tabular} Zhuo et al.: PTPN11 Polymorphisms and GC}

Table 8. The conditional logistic regression analysis on interaction between PTPN11 SNPs (i.e. rs12229892 and rs2301756) and intake of processed food

\begin{tabular}{lllllllll}
\hline Genotype & Intake of processed food & Case $(\mathrm{n}, \%)$ & Control $(\mathrm{n}, \%)$ & OR & $95 \% \mathrm{CI}$ & $\beta e$ & $\beta e g$ & $\gamma$ \\
\hline rs12229892 & & & & & & & & \\
GG & No & $35(8.14 \%)$ & $169(17.60 \%)$ & & & & & \\
GA & No & $85(19.77 \%)$ & $238(24.79 \%)$ & 1.72 & $1.11-2.68$ & & \\
AA & No & $24(5.58 \%)$ & $95(9.89 \%)$ & 1.22 & $0.68-2.17$ & & \\
GG & Yes & $69(16.05 \%)$ & $155(16.15 \%)$ & 2.15 & $1.36-3.41$ & 0.77 & & \\
GA & Yes & $170(39.53 \%)$ & $217(22.60 \%)$ & 3.78 & $2.50-5.73$ & 0.77 & 1.33 & 1.74 \\
AA & Yes & $47(10.93 \%)$ & $86(8.96 \%)$ & 2.64 & $1.59-4.39$ & 0.77 & 0.97 & 1.27 \\
rs2301756 & & & & & & & & \\
GG & No & $98(22.79 \%)$ & $332(34.58 \%)$ & & & & & \\
GA & No & $41(9.53 \%)$ & $136(14.17 \%)$ & 1.02 & $0.67-1.55$ & & & \\
AA & No & $4(0.93 \%)$ & $34(3.54 \%)$ & 0.40 & $0.14-1.51$ & & & \\
GG & Yes & $197(45.81 \%)$ & $302(31.46 \%)$ & 2.21 & $1.66-2.95$ & 0.79 & & \\
GA & Yes & $82(19.07 \%)$ & $125(13.02 \%)$ & 2.22 & $1.55-3.18$ & 0.79 & 0.80 & 1.01 \\
AA & Yes & $8(1.87 \%)$ & $31(3.23 \%)$ & 0.87 & $0.39-1.96$ & 0.79 & -0.14 & -0.18 \\
\hline
\end{tabular}

Table 9. The conditional logistic regression analysis on interaction between PTPN11 SNPs (i.e. rs12229892 and rs2301756) and H. pylori infection

\begin{tabular}{lllllllll}
\hline Genotype & H. pylori infection & Case $(\mathrm{n}, \%)$ & Control $(\mathrm{n}, \%)$ & OR & $95 \% \mathrm{CI}$ & $\beta e$ & $\beta e g$ & $\gamma$ \\
\hline rs12229892 & & & & & & & & \\
GG & No & $31(7.21 \%)$ & $165(17.19 \%)$ & & & & & \\
GA & No & $77(17.91 \%)$ & $231(24.06 \%)$ & 1.77 & $1.12-2.81$ & & & \\
AA & No & $21(4.88 \%)$ & $92(9.58 \%)$ & 1.22 & $0.66-2.24$ & & & \\
GG & Yes & $73(16.98 \%)$ & $159(16.56 \%)$ & 2.44 & $1.52-3.92$ & 0.89 & & \\
GA & Yes & $178(41.40 \%)$ & $224(23.33 \%)$ & 4.23 & $2.75-6.51$ & 0.89 & 1.44 & 1.62 \\
AA & Yes & $50(11.62 \%)$ & $89(9.27 \%)$ & 2.99 & $1.78-5.02$ & 0.89 & 1.10 & 1.23 \\
rs2301756 & & & & & & & & \\
GG & No & $89(20.70 \%)$ & $322(33.54 \%)$ & & & & & \\
GA & No & $37(8.60 \%)$ & $133(13.85 \%)$ & 1.01 & $0.65-1.55$ & & & \\
AA & No & $4(0.93 \%)$ & $33(3.44 \%)$ & 0.44 & $0.15-1.27$ & & & \\
GG & Yes & $206(47.91 \%)$ & $312(32.50 \%)$ & 2.39 & $1.78-3.20$ & 0.87 & & \\
GA & Yes & $86(20.00 \%)$ & $128(13.33 \%)$ & 2.43 & $1.70-3.49$ & 0.87 & 0.89 & 1.02 \\
AA & Yes & $8(1.86 \%)$ & $32(3.34 \%)$ & 0.90 & $0.40-2.03$ & 0.87 & -0.11 & -0.12 \\
\hline
\end{tabular}

Correlation between combined effects of PTPN11 polymorphisms (rs12229892 and rs2301756) and susceptibility to GC

Compared with other genotype combinations (Table 5), homozygote GG of rs 12229892 coupled with homozygote GG or AA of rs2301756 seemed to confer protective effects on GC risk $(\mathrm{OR}=0.69,95 \% \mathrm{CI}=0.51-0.63, P=0.014 ; \mathrm{OR}=0.30,95 \% \mathrm{CI}=0.09-1.01, P=0.039)$. On the contrary, heterozygote GA of rs12229892 combined with either GA or GG genotype of rs 2301756 could be more frequently found among GC populations with their ORs of 1.38 $(95 \% \mathrm{CI}=1.01-1.89, P=0.045)$ and $1.50(95 \% \mathrm{CI}=1.19-1.90, P<0.001)$, respectively.

Interaction between PTPN11 polymorphisms (rs12229892 and rs2301756) and environmental hazards (i.e. smoking status, family history, intake of processed food and H. pylori infection)

Even though smoking $\left(\mathrm{OR}_{\mathrm{e}}=1.42\right)$ or homozygote AA of rs12229892 $\left(\mathrm{OR}_{\mathrm{g}}=1.21\right)$ alone might not significantly serve as predisposing factors for GC development, their 


\section{Cellular Physiology Cell Physiol Biochem 2016;39:1537-1552 \begin{tabular}{ll|l} 
DOI: 10.1159/000447856 & ( 2016 The Author(s). Published by S. Karger AG, Basel
\end{tabular} Zhuo et al.: PTPN11 Polymorphisms and GC}

Table 10. Association of variables with GC patients' overall survival

\begin{tabular}{|c|c|c|c|c|c|c|}
\hline \multirow{2}{*}{ Variable } & \multicolumn{2}{|c|}{ Univariate analysis } & \multicolumn{4}{|c|}{ Multivariate analysis } \\
\hline & $\mathrm{OR}$ & $95 \% \mathrm{Cl}$ & $P$ value & $\mathrm{OR}$ & $95 \% \mathrm{CI}$ & $P$ value \\
\hline \multicolumn{7}{|l|}{ Pathological subtype } \\
\hline Adenocarcinoma & Reference & & & & & \\
\hline Mucoid carcinoma & 0.68 & $0.46-1.00$ & 0.053 & 0.73 & $0.49-1.08$ & 0.118 \\
\hline Signet-ring cell carcinoma & 0.86 & $0.61-1.21$ & 0.380 & 0.81 & $0.58-1.15$ & 0.246 \\
\hline Others & 1.22 & $0.86-1.72$ & 0.262 & 1.23 & $0.86-1.76$ & 0.268 \\
\hline \multicolumn{7}{|l|}{ Differentiation degree } \\
\hline Well differentiated & Reference & & & & & \\
\hline Moderately differentiated & 16.42 & $2.92-117.55$ & 0.005 & 10.86 & $1.50-78.36$ & 0.018 \\
\hline Poorly differentiated & 53.87 & $7.53-385.26$ & $<0.001$ & 26.35 & $3.64-190.60$ & 0.001 \\
\hline Others & 27.51 & $3.71-204.26$ & 0.001 & 15.98 & $2.12-120.62$ & 0.007 \\
\hline \multicolumn{7}{|l|}{ Clinical stages } \\
\hline IIIA & Reference & & & & & \\
\hline IIIB & 2.62 & $0.93-7.39$ & 0.069 & 0.80 & $0.28-2.29$ & 0.676 \\
\hline IIIC & 5.49 & $2.04-14.78$ & 0.001 & 1.31 & $0.48-3.57$ & 0.604 \\
\hline IV & 6.02 & $2.22-16.32$ & $<0.001$ & 1.59 & $0.58-4.36$ & 0.367 \\
\hline \multicolumn{7}{|l|}{ Axillary lymph node metastasis } \\
\hline N0-1 & Reference & & & & & \\
\hline $\mathrm{N} 2-3$ & 4.38 & $3.07-6.26$ & $<0.001$ & 2.60 & $1.80-3.77$ & $<0.001$ \\
\hline \multicolumn{7}{|l|}{ CEA } \\
\hline$<5 \mathrm{ng} / \mathrm{mL}$ & Reference & & & & & \\
\hline$\geq 5 \mathrm{ng} / \mathrm{mL}$ & 1.30 & $1.05-1.60$ & 0.014 & 1.14 & $0.92-1.41$ & 0.222 \\
\hline \multicolumn{7}{|l|}{ rs12229892 } \\
\hline GG & Reference & & & & & \\
\hline $\mathrm{GA}+\mathrm{AA}$ & 2.47 & $1.88-3.25$ & $<0.001$ & 1.91 & $1.44-2.53$ & $<0.001$ \\
\hline \multicolumn{7}{|l|}{ rs2301756 } \\
\hline GG & Reference & & & & & \\
\hline $\mathrm{GA}+\mathrm{AA}$ & 1.91 & $1.54-2.37$ & $<0.001$ & 0.57 & $0.44-0.72$ & $<0.001$ \\
\hline
\end{tabular}

additive effects were strikingly correlated with increased $\mathrm{GC}$ risk $\left(\mathrm{OR}_{\mathrm{eg}}=1.76,95 \% \mathrm{CI}=\right.$ 1.07-2.89) (Tables 6-9). Since their $\gamma$ value was equivalent to $1.61(>1), \stackrel{\mathrm{eg}}{\mathrm{r}} 12229892 \mathrm{AA}$ and smoking were inferred to be positively interacted in driving occurrence of GC, indicating their mutually amplified effects in aggravating GC risk. Moreover, as 1.76 was more than the product of 1.42 and 1.21, the interaction mechanism regarding AA of rs12229892 and smoking was considered as the super-multiplicative model. Similarly, the underlying mechanism concerning rs12229892 AA and three other environmental factors were also confirmed as super-multiplicative model (family history, $\mathrm{OR}_{\mathrm{eg}} 2.36>\mathrm{OR}_{\mathrm{e}} 1.92 \times \mathrm{OR}_{\mathrm{g}} 1.22$; intake of processed food, $\mathrm{OR}_{\mathrm{eg}} 2.64>\mathrm{OR}_{\mathrm{e}} 2.15 \times \mathrm{OR}_{\mathrm{g}} 1.22 ; \mathrm{H}$. pylori infection, $\mathrm{OR}_{\mathrm{eg}} 2.999^{\mathrm{g}}>\mathrm{OR}_{\mathrm{e}}$ $2.44 \times \mathrm{OR}_{\mathrm{g}} 1.22$ ).

As far as rs2301756 was concerned, smoking $\left(\mathrm{OR}_{\mathrm{e}}=1.41,95 \% \mathrm{CI}=1.07-1.86\right)$ alone was closely related with incremental susceptibility to GC, but the co-existence of smoking and homozygote AA made this tight correlation less distinct and even the harmful tendency was reversed to a protective trend $\left(\mathrm{OR}_{\mathrm{eg}}=0.53\right)$. Taking $\gamma=-1.84(<0)$ and $\beta_{\mathrm{e}}=0.34(>0)$ into account, smoking and AA genotype interacted negatively concerning their combined effects on GC risk and homozygote AA could largely inhibit incidence of GC. Besides, the inequality $\left(\mathrm{OR}_{\mathrm{eg}} 0.53<\mathrm{OR}_{\mathrm{e}} 1.41 \times \mathrm{OR}_{\mathrm{g}} 0.42\right)$ implied that the reciprocal action of smoking and AA genotype tallied with the sub-multiplicative model. Analogously, the joint impacts of 


\section{Cellular Physiology Cell Physiol Biochem 2016;39:1537-1552 \begin{tabular}{ll|l} 
aOI: 10.1159/000447856 & $\begin{array}{l}\text { @ 2016 The Author(s). Published by S. Karger AG, Basel } \\
\text { www.karger.com/cpb }\end{array}$ \\
\hline
\end{tabular}

Fig. 1. Kaplan-Meier survival curve relevant to gastric cancer patients with various differentiation degrees (i.e. well differentiation, moderate differentiation, poor differentiation and others) $(P<0.001)$.

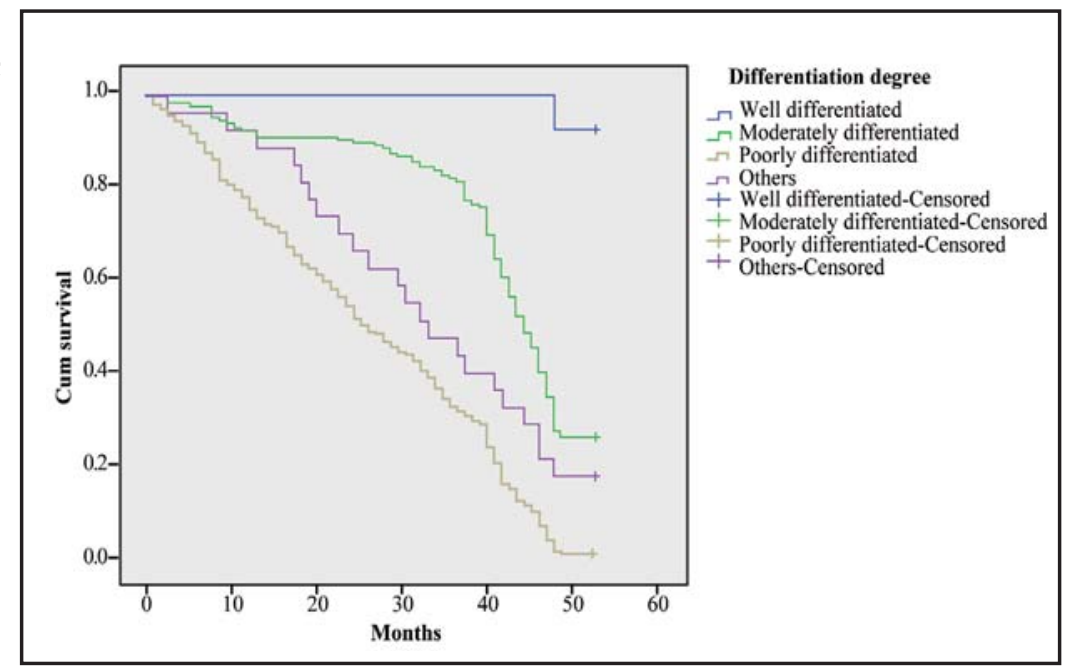

Fig. 2. Kaplan-Meier survival curve relevant to gastric cancer patients with distinct degrees of axillary lymph node metastasis (i.e. N0-1 and N23) $(P<0.001)$.

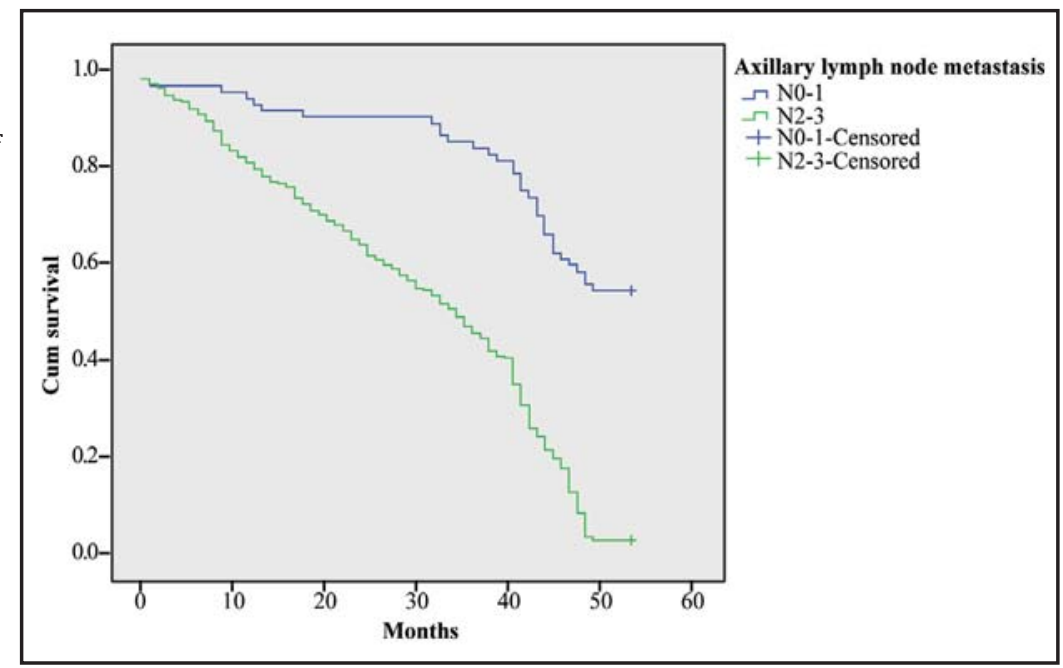

family history/intake of processed food/H. pylori infection and AA genotype on GC risk were indicated as the sub-multiplicative model (family history, $\mathrm{OR}_{\mathrm{eg}} 0.74<\mathrm{OR}_{\mathrm{e}} 1.96 \times \mathrm{OR}_{\mathrm{g}} 0.40$; intake of processed food, $\mathrm{OR}_{\mathrm{eg}} 0.87<\mathrm{OR}_{\mathrm{e}} 2.21 \times \mathrm{OR}_{\mathrm{g}} 0.40 ; \mathrm{H}$. pylori infection, $\mathrm{OR}_{\mathrm{eg}} 0.90^{\mathrm{g}}<\mathrm{OR}_{\mathrm{e}}$ $\left.2.39 \times 0 R_{g} 0.44\right)$. The corresponding $\gamma$ values (all $<0$ ) and $\beta_{e}$ values (all $>0$ ) also showed that homozygote AA could greatly compete against the effects imposed by family history/intake of processed food/H. pylori infection on GC development.

\section{Association between PTPN11 polymorphism and prognosis of GC}

Totally 430 GC cases were followed up with the median survival time of 46 months, and 67 cases $(15.58 \%)$ survived to the end. As was displayed in Table 10, type of GC (i.e. adenocarcinoma, mucoid carcinoma, signet-ring cell carcinoma and others) seemed not be the determining factor of OS through judgement of either univariate or multivariate analyses (all $P>0.05$ ). Nonetheless, poorly-differentiated GC was obviously associated with the least survival time of investigated subjects (Long-Rank $P<0.001$ ) (Fig. 1) and moderatelydifferentiated GCs might anticipate shorter life span than well-differentiated GCs regardless of pathological subtype, clinical stage, axillary lymph node metastasis and other elements (OR $=10.86,95 \% \mathrm{CI}=1.50-78.36, P=0.018)$. Additionally, axillary lymph node metastasis (N2-3 vs. N0-1), rs12229892 (GA +AA vs. GG) and rs2301756 (GA +AA vs. GG) were also independently associated with dramatically altered mortality of GC patients based on multivariate analyses $(\mathrm{OR}=2.60,95 \% \mathrm{CI}=1.80-3.77, P<0.001 ; \mathrm{OR}=1.91,95 \% \mathrm{CI}=1.44$ $2.53, P<0.001 ; \mathrm{OR}=0.57,95 \% \mathrm{CI}=0.44-0.72, P<0.001$ ) (Fig. $2-4$ ). 


\section{Cellular Physiology}

Cell Physiol Biochem 2016;39:1537-1552

\begin{tabular}{l|l}
\hline DOI: $10.1159 / 000447856$ & (C) 2016 The Author(s). Published by S. Karger AG, Base
\end{tabular} and Biochemistry Published online: September 12, 2016 www.karger.com/cpb

Fig. 3. Kaplan-Meier survival curve of gastric cancer patients carrying diverse genotypes (i.e. GG, GA and AA) of rs12229892 $(P<0.001)$.

Fig. 4. Kaplan-Meier survival curve of gastric cancer patients carrying diverse genotypes (i.e. GG, GA and AA) of rs2301756 $(P<0.001)$.
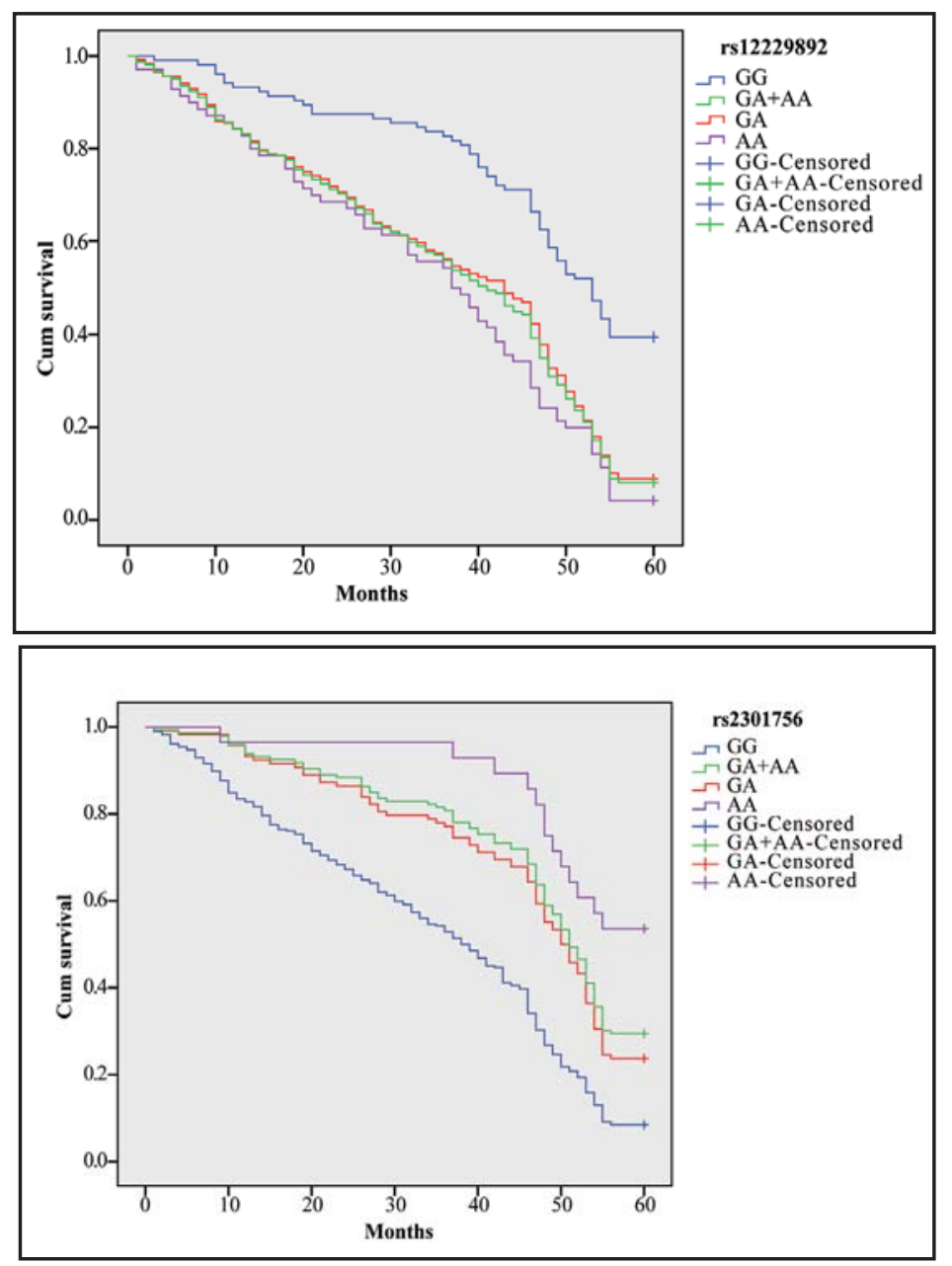

Fig. 5. Kaplan-Meier survival curve relevant to gastric cancer patients in various clinical stages (i.e. IIIA, IIIB, IIIC and IV stages) $(P<$ 0.001).

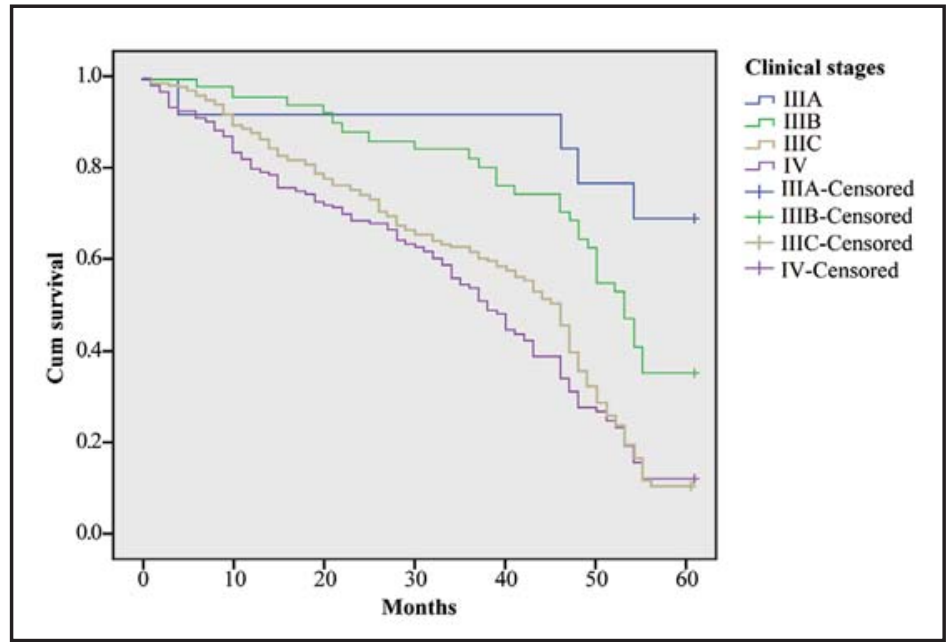

Furthermore, comparisons of OS among IIIA, IIIB, IIIC and IV stages indicated that the more advanced the clinical stage, the higher death rate of GC patients (Long-Rank $P<0.001$ ) (Fig. 5), though the significant tendency did not persist after adjustment of multivariate analyses (all $P>0.05$ ). Similar circumstances also appeared on the indicator of CEA, which demonstrated that GC cases with in vivo CEA content of $\geq 5 \mathrm{ng} / \mathrm{mL}$ were more likely to obtain 


\section{Cellular Physiology and Biochemistry}

Cell Physiol Biochem 2016;39:1537-1552

Fig. 6. Kaplan-Meier survival curve relevant to gastric cancer patients with distinct carcinoembryonic antigen (CEA) levels (i.e. $<5$ $\mathrm{ng} / \mathrm{ml}$ and $\geq 5 \mathrm{ng} / \mathrm{ml})(P=0.012)$.

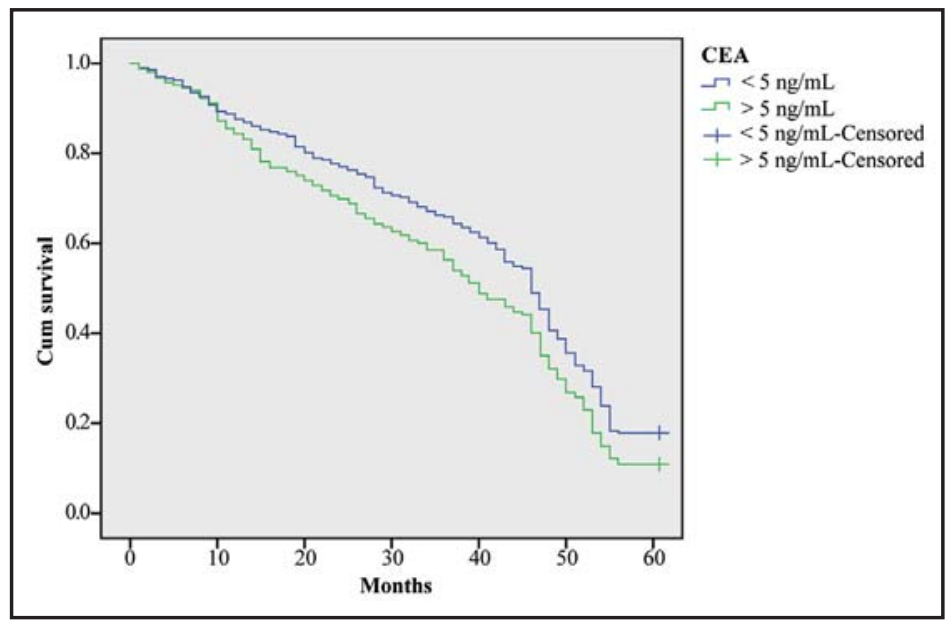

pessimistic prognosis than those carrying CEA content of $<5 \mathrm{ng} / \mathrm{mL}$ (Long-Rank $P=0.012$ ) (Fig. 6). However, the overwhelming differentiation $(\mathrm{OR}=1.30,95 \% \mathrm{CI}=1.05-1.60, P<$ $0.014)$ was not replicated after eliminating impacts of extra parameters $(P=0.106$; Table 10).

\section{Discussion}

Human GC is still the primary lethal cancer among all malignant neoplasms despite current therapeutic approaches including surgery, radiation and chemotherapy have improved the overall survival status of GC patients. Therefore, the association of PTPN11 with GC risk was explored in this study to find possible treatment targets for GC, which indicated that remarkable differences in genotype and allelic distributions existed between case and control groups for rs12229892 and rs2301756. In addition, both 2-cycle and 4-cycle chemotherapy were more effective in individuals with mutation gene A (GA+AA) of rs2301756, and genotypes GA/AA of rs2301756 are independent protective factors for GC prognosis.

Individuals infected by $H$. pylori, which carries CagA, are associated with significantly higher risk of GC [21]. Particularly, once CagA bound to SHP2, the encoded protein of PTPN11, cell motility along with a highly stretched cell shape were elevated [22], which actually counted much in forming gastric carcinoma [23]. Several studies conducted in Japanese populations have reported a correlation between PTPN11 rs2301756 in the intron 3 and gastric atrophy identified in subjects with $H$. pylori infections, providing evidence that Japanese individuals who have A allele of the PTPN11 rs2301756 were associated with a significantly lower risk of severe gastric atrophy which is a precursory phase of GC [12]. The present study also considered G allele of PTPN11 rs2301756 as one susceptible element for GC when compared with A allele. Conversely, a case-control study among the Uzbekistan population indicated that $\mathrm{G}$ allele of PTPN11 rs2301756 could augment the onset risk of gastric atrophy [24]. The inconsistency might be ascribed to distinctions in the genetic distribution among Japanese, Chinese and Uzbeks. It is hypothesized that rs2301756 might be involved in shaping mRNA splicing variants of PTPN11, owing to the fact that this SNP is situated merely 223 base pairs upstream from exon 4, which partly determines encoding of C-terminal src homology 2 (SH2) domain of SHP-2 [12]. Of note, it is the SH2 domain that binds to tyrosine phosphorylated CagA, in which way conformational alterations of SHP-2 are induced and SHP-2 phosphatase is thus activated $[25,26]$. Hence, it is quite likely that homozygote AA or allele A of rs2301756 is crucial for prevention of GC development with homozygote GG or allele G as respective controls. Meanwhile, rs12229892 variant A-allele was previously revealed to be associated with a reduced risk of GC in subjects without $H$. pylori infection but an increased risk of GC in individuals with $H$. pylori infection [27]. The current 


\section{Cellular Physiology Cell Physiol Biochem 2016;39:1537-1552 \\ \begin{tabular}{ll|l} 
and Biochemistry & $\begin{array}{l}\text { DOI: 10.1159/000447856 } \\
\text { Published online: September 12, } 2016\end{array}$ & $\begin{array}{l}\text { ( ) 2016 The Author(s). Published by S. Karger AG, Basel } \\
\text { www.karger.com/cpb }\end{array}$ \\
\hline
\end{tabular} \\ Zhuo et al.: PTPN11 Polymorphisms and GC}

study also certified the association of rs12229892 with GC, yet the underlying mechanism remained vague. Accordingly, two G/A SNPs of PTPN11 (rs2301756 and rs12229892) have been considered as candidate polymorphisms for this Chinese population [18, 24]. However, both functional analysis and larger-scale studies should be undertaken to confirm the relationship between PTPN11 polymorphisms and GC.

Besides, cisplatin and 5-fluorouracil (5-Fu) were essential drugs in the process of applying chemotherapies for gastrointestinal tumors due to their capacities of inhibiting cell proliferation [28]. Specifically, the metabolites of 5-Fu mainly interfered with DNA synthesis through acting on enzymes that were necessary for DNA synthesis to form stable covalent complexes, while cisplatin principally formed intra-chain/inter-chain DNA adducts to hinder DNA replication $[29,30]$. In contrast, SHP2 usually served to enhance cell proliferation and motility via binding to multiple molecules, so as to modify certain pathways in numerous tumors such as laryngeal and breast cancer [31, 32]. For instance, SHP-2 could interact with scaffolding/adaptor protein (i.e. Gab2) to accelerate hyperprolifertaion of mammary cells [33]. In addition, SHP2 was deemed to basally regulate Jak/STAT signaling induced by IL-6, which commonly functioned in cancer cells [34, 35]. SHP2 also mediates GC progression under the influence of PTEN/AKT signaling and promotes growth of laryngeal cancer through the Ras/Raf/Mek/Erk pathway [36]. To sum up, DNA synthesis and replications affected by SHP-2 might be accelerated before they were blocked by cisplatin and 5-Fu. As a result, it was inferred that GC patients with dysfunctional SHP-2 would suffer from more undesirable prognosis than those with normal SHP-2, since the degree of identical cisplatin and 5-Fu to restrain in-vivo tumor proliferation for GC patients with aberrant SHP-2 appeared lower than that for GC patients with normal SHP-2. Therefore, under the effects of SHP-2-induced cell hyperproliferation, it was acceptable that GC patients carrying homozygote GG of rs2301756 were associated with poorer short-term efficacies after chemotherapies than those with GA/ AA genotypes. However, whether SHP-2 directly acted on target DNA still demanded further investigations.

In addition, smoking and intake of processed food were eventually concluded to be two independent risk factors for GC development. The explanations might be that smoking could trigger intestinal metaplasia and mal-development of gastric mucosa, which would progressively develop into GC [37]. To be specific, risks of intestinal metaplasia and maldeveloped gastric mucosa would be increased to 118 and 316 folds, respectively, for subjects smoking > 10 cigarettes per day when compared with non-smokers [38]. Moreover, smoking was deduced to genetically incapacitate organisms from cancer-suppression by bringing down active P53 contents and enhancing c-myc expressions [39]. Similarly, processed food also injured gastric mucosa and even advanced genetic mutations that could be relevant to $\mathrm{GC}$, but based on a different mechanism that nitrates and nitrites within processed food could be transformed to harmful N-nitrosamides with aid of stomach bacterial actions [40, 41].

Despite the above results, several defects still existed. First of all, the studied populations were of one single ethnic background (i.e. Han Chinese), so the conclusion was less convincible if it was generalized to other ethnicities. Besides, the selected SNPs were not full-scale enough and extra potentially functional SNPs could still not be identified. Moreover, though the sample size seemed considerable, a larger sample size would be more reliable. Finally, finite elements relevant to GC prognosis were investigated, for instance, psychological pressure could indeed aggravate GC prognosis for that patients with advanced GC suffered from more severe anxiety and depression than those at the initial stage of GC [42]. Hence, to confirm PTPN11 genetic polymorphisms as independent factors for undesirable GC prognosis, the effects of mental pressure should be excluded.

In summary, this study conducted on a Chinese population concluded that rs2301756 in the PTPN11 which encodes SHP-2 was associated with a reduced risk of GC. Since PTPN11 polymorphism in the intron 3 region is a sensitive indicator which evaluates the effect of chemotherapy on GC, it may be considered as an independent predictor of GC prognosis. As a result, this study may provide additional information for both GC diagnosis and prognosis. 


\section{Cellular Physiology Cell Physiol Biochem 2016;39:1537-1552

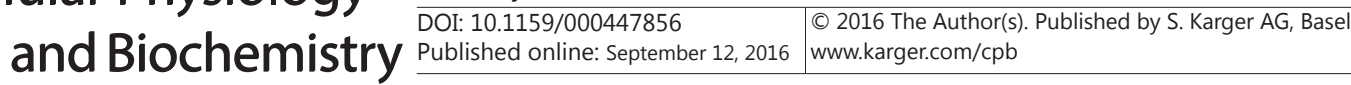 Zhuo et al.: PTPN11 Polymorphisms and GC}

\section{Abbreviations}

GC (gastric cancer); PCR-RFLP (polymerase chain reaction-restriction fragment length polymorphism); ORs (Odds ratios); CIs (confidence intervals); H. pylori (Helicobacter pylori); SHP-2 (signaling protein); SNPs (single-nucleotide polymorphisms); EDTA (ethylene diamine tetraacetic acid); RECIST (response evaluation criteria in solid tumors); CR (complete sresponse); PR (partial response); SD (stable disease); PD (progressive disease); HWE (Hardy-Weinberg equilibrium); GEI (gene-environment interaction).

\section{Acknowledgements}

This work was supported by grants from the Science and Technology Fund of Tianjin Health Bureau (2014KR02 to Chuanjun Zhuo), the China Postdoctoral Science Foundation (2012M 520585 to Chuanjun Zhuo), the Found of Wenzhou Health Bureau (2013B28 to Guangdong Chen), the Foundation of Jiangsu Haosen Pharmaceutical Co., Ltd (to Chuanjun Zhuo), the Foundation of Rongchang Pharmaceutical Co., Ltd (to Chuanjun Zhuo), the Foundation of Hainan Li Ou Pharmaceutical Co., Ltd (to Chuanjun Zhuo) and the Foundation of Xuzhou Enhua Pharmaceutical Co., Ltd (to Chuanjun Zhuo).

\section{Disclosure Statement}

The authors have no conflicts of interest to disclose.

\section{References}

1 Torre LA, Bray F, Siegel RL, Ferlay J, Lortet-Tieulent J, Jemal A: Global cancer statistics, 2012. CA Cancer J Clin 2015;65:87-108.

2 Jiang H, Wang H, Ge F, Wu L, Wang X, Chen S: The Functional Variant in the 3'UTR of IGF1 with the Risk of Gastric Cancer in a Chinese Population. Cell Physiol Biochem 2015;36:884-892.

3 Hatakeyama M: Helicobacter pylori and gastric carcinogenesis. J Gastroenterol 2009;44:239-248.

4 Kabir S: Effect of Helicobacter pylori eradication on incidence of gastric cancer in human and animal models: underlying biochemical and molecular events. Helicobacter 2009;14:159-171.

5 Lochhead P, El-Omar EM: Helicobacter pylori infection and gastric cancer. Best Pract Res Clin Gastroenterol 2007;21:281-297.

6 Amieva MR, El-Omar EM: Host-bacterial interactions in Helicobacter pylori infection. Gastroenterology 2008;134:306-323.

7 Hishida A, Matsuo K, Goto Y, Hamajima N: Genetic predisposition to Helicobacter pylori-induced gastric precancerous conditions. World J Gastrointest Oncol 2010;2:369-379.

8 Snaith A, El-Omar EM: Helicobacter pylori: host genetics and disease outcomes. Expert Rev Gastroenterol Hepatol 2008;2:577-585.

9 Yamazaki S, Yamakawa A, Ito Y, Ohtani M, Higashi H, Hatakeyama M, Azuma T: The CagA protein of Helicobacter pylori is translocated into epithelial cells and binds to SHP-2 in human gastric mucosa. J Infect Dis 2003; 187:334-337.

10 Jiang J, Jia ZF, Kong F, Jin MS, Wang YP, Tian S, Suo J, Cao X: Association of polymorphism of PTPN 11 encoding SHP-2 with gastric atrophy but not gastric cancer in Helicobacter pylori seropositive Chinese population. BMC Gastroenterol 2012;12:89.

11 Segal ED, Cha J, Lo J, Falkow S, Tompkins LS: Altered states: involvement of phosphorylated CagA in the induction of host cellular growth changes by Helicobacter pylori. Proc Natl Acad Sci U S A 1999;96:1455914564. 


\section{Cellular Physiology Cell Physiol Biochem 2016;39:1537-1552 \begin{tabular}{rl|l} 
and Biochemistry $10.1159 / 000447856$ & $\begin{array}{l}\text { @ 2016 The Author(s). Published by S. Karger AG, Basel } \\
\text { www.karger.com/cpb }\end{array}$ \\
\hline
\end{tabular} Zhuo et al.: PTPN11 Polymorphisms and GC}

12 Hishida A, Matsuo K, Goto Y, Naito M, Wakai K, Tajima K, Hamajima N: Associations of a PTPN11 G/A polymorphism at intron 3 with Helicobactor pylori seropositivity, gastric atrophy and gastric cancer in Japanese. BMC Gastroenterol 2009;9:51.

13 Kim JS, Shin OR, Kim HK, Cho YS, An CH, Lim KW, Kim SS: Overexpression of protein phosphatase nonreceptor type 11 (PTPN11) in gastric carcinomas. Dig Dis Sci 2010;55:1565-1569.

14 Santoro R, Carboni F, Lepiane P, Ettorre GM, Santoro E: Clinicopathological features and prognosis of gastric cancer in young European adults. Br J Surg 2007;94:737-742.

15 Cunningham D, Allum WH, Stenning SP, Thompson JN, Van de Velde CJ, Nicolson M, Scarffe JH, Lofts FJ, Falk SJ, Iveson TJ, Smith DB, Langley RE, Verma M, Weeden S, Chua YJ, Participants MT: Perioperative chemotherapy versus surgery alone for resectable gastroesophageal cancer. N Engl J Med 2006;355:11-20.

16 Li QF, Yao RY, Liu KW, Lv HY, Jiang T, Liang J: Genetic polymorphism of GSTP1: prediction of clinical outcome to oxaliplatin/5-FU-based chemotherapy in advanced gastric cancer. J Korean Med Sci 2010;25:846-852.

17 Oh SY, Kwon HC, Kim SH, Lee S, Lee JH, Hwang JA, Hong SH, Graves CA, Camphausen K, Kim HJ, Lee YS: The relationship of vascular endothelial growth factor gene polymorphisms and clinical outcome in advanced gastric cancer patients treated with FOLFOX: VEGF polymorphism in gastric cancer. BMC Cancer 2013;13:43.

18 Goto Y, Ando T, Yamamoto K, Tamakoshi A, El-Omar E, Goto H, Hamajima N: Association between serum pepsinogens and polymorphismof PTPN11 encoding SHP-2 among Helicobacter pylori seropositive Japanese. Int J Cancer 2006;118:203-208.

19 Shostak S: Locating gene-environment interaction: at the intersections of genetics and public health. Soc Sci Med 2003;56:2327-2342.

20 Sun JB, Raghavan S, Sjoling A, Lundin S, Holmgren J: Oral tolerance induction with antigen conjugated to cholera toxin B subunit generates both Foxp3+CD25+ and Foxp3-CD25- CD4+ regulatory T cells. J Immunol 2006;177:7634-7644.

21 Meng F, Zhao X, Zhang S: Expression and significance of SHP-2 in human papillomavirus infected cervical cancer. J Huazhong Univ Sci Technolog Med Sci 2012;32:247-251.

22 Azuma T, Yamazaki S, Yamakawa A, Ohtani M, Muramatsu A, Suto H, Ito Y, Dojo M, Yamazaki Y, Kuriyama M, Keida Y, Higashi H, Hatakeyama M: Association between diversity in the Src homology 2 domain-containing tyrosine phosphatase binding site of Helicobacter pylori CagA protein and gastric atrophy and cancer. J Infect Dis 2004;189:820-827.

23 Kurashima Y, Murata-Kamiya N, Kikuchi K, Higashi H, Azuma T, Kondo S, Hatakeyama M: Deregulation of beta-catenin signal by Helicobacter pylori CagA requires the CagA-multimerization sequence. Int J Cancer 2008;122:823-831.

24 Hamajima N, Rahimov B, Malikov Y, Abdiev S, Ahn KS, Bahramov S, Kawai S, Nishio K, Naito M, Goto Y: Associations between a PTPN11 polymorphism and gastric atrophy--opposite in Uzbekistan to that in Japan. Asian Pac J Cancer Prev 2008;9:217-220.

25 Higashi H, Tsutsumi R, Fujita A, Yamazaki S, Asaka M, Azuma T, Hatakeyama M: Biological activity of the Helicobacter pylori virulence factor CagA is determined by variation in the tyrosine phosphorylation sites. Proc Natl Acad Sci U S A 2002;99:14428-14433.

26 Hatakeyama M: Helicobacter pylori CagA -- a bacterial intruder conspiring gastric carcinogenesis. Int J Cancer 2006;119:1217-1223.

27 He C, Tu H, Sun L, Xu Q Gong Y, Jing J, Dong N, Yuan Y: SNP interactions of Helicobacter pylori-related host genes PGC, PTPN11, IL1B, and TLR4 in susceptibility to gastric carcinogenesis. Oncotarget 2015;6:1901719026.

28 Matsuhashi N, Saio M, Matsuo A, Sugiyama Y, Saji S: Expression of p53 protein as a predictor of the response to 5-fluorouracil and cisplatin chemotherapy in human gastrointestinal cancer cell lines evaluated with apoptosis by use of thin layer collagen gel. Int J Oncol 2004;24:807-813.

29 Li J, Wang SX: Synergistic enhancement of the antitumor activity of 5-fluorouracil by bornyl acetate in SGC7901 human gastric cancer cells and the determination of the underlying mechanism of action. J BUON 2016;21:108-117.

30 Gazdar AF: DNA repair and survival in lung cancer--the two faces of Janus. N Engl J Med 2007;356:771-773.

31 Gu J, Han T, Ma RH, Zhu YL, Jia YN, Du JJ, Chen Y, Jiang XJ, Xie XD, Guo X: SHP2 promotes laryngeal cancer growth through the Ras/Raf/Mek/Erk pathway and serves as a prognostic indicator for laryngeal cancer. Int J Oncol 2014;44:481-490. 


\section{Cellular Physiology Cell Physiol Biochem 2016;39:1537-1552 \begin{tabular}{ll|l}
\cline { 2 - 2 } DOI: 10.1159/000447856 & () 2016 The Author(s). Published by S. Karger AG, Basel
\end{tabular} and Biochemistry Published online: September 12, 2016 www.karger.com/cpb}

Zhuo et al.: PTPN11 Polymorphisms and GC

32 Hu Z, Fang H, Wang X, Chen D, Chen Z, Wang S: Overexpression of SHP2 tyrosine phosphatase promotes the tumorigenesis of breast carcinoma. Oncol Rep 2014;32:205-212.

33 Bentires-Alj M, Gil SG, Chan R, Wang ZC, Wang Y, Imanaka N, Harris LN, Richardson A, Neel BG, Gu H: A role for the scaffolding adapter GAB2 in breast cancer. Nat Med 2006;12:114-121.

34 Dittrich A, Quaiser T, Khouri C, Gortz D, Monnigmann M, Schaper F: Model-driven experimental analysis of the function of SHP-2 in IL-6-induced Jak/STAT signaling. Mol Biosyst 2012;8:2119-2134.

35 Hubackova S, Novakova Z, Krejcikova K, Kosar M, Dobrovolna J, Duskova P, Hanzlikova H, Vancurova M, Barath P, Bartek J, Hodny Z: Regulation of the PML tumor suppressor in drug-induced senescence of human normal and cancer cells by JAK/STAT-mediated signaling. Cell Cycle 2010;9:3085-3099.

36 Tseng PC, Huang WC, Chen CL, Sheu BS, Shan YS, Tsai CC, Wang CY, Chen SO, Hsieh CY, Lin CF: Regulation of SHP2 by PTEN/AKT/GSK-3beta signaling facilitates IFN-gamma resistance in hyperproliferating gastric cancer. Immunobiology 2012;217:926-934.

37 Amieva MR, Vogelmann R, Covacci A, Tompkins LS, Nelson WJ, Falkow S: Disruption of the epithelial apicaljunctional complex by Helicobacter pylori CagA. Science 2003;300:1430-1434.

38 Wong BC, Ching CK, Lam SK: Helicobacter pylori infection and gastric cancer. Hong Kong Med J 1999;5:175-179.

39 Wei J, O'Brien D, Vilgelm A, Piazuelo MB, Correa P, Washington MK, El-Rifai W, Peek RM, Zaika A: Interaction of Helicobacter pylori with gastric epithelial cells is mediated by the p53 protein family. Gastroenterology 2008;134:1412-1423.

40 Lin SH, Li YH, Leung K, Huang CY, Wang XR: Salt processed food and gastric cancer in a Chinese population. Asian Pac J Cancer Prev 2014;15:5293-5298.

41 Weerasooriya S, Jasti VP, Bose A, Spratt TE, Basu AK: Roles of translesion synthesis DNA polymerases in the potent mutagenicity of tobacco-specific nitrosamine-derived 02-alkylthymidines in human cells. DNA Repair (Amst) 2015;35:63-70.

42 Kang JI, Chung HC, Jeung HC, Kim SJ, An SK, Namkoong K: FKBP5 polymorphisms as vulnerability to anxiety and depression in patients with advanced gastric cancer: a controlled and prospective study. Psychoneuroendocrinology 2012;37:1569-1576. 